\title{
Evaluación socioeconómica de disposición a pagar por aprovechamiento y preservación del recurso hídrico en Cubijíes
}

\author{
Socioeconomic evaluation of willingness to pay for use and preservation of \\ water resources in Cubijíes
}

Sofía Carolina Godoy Ponce. ${ }^{1}$, Andrés Agustín Beltrán Dávalos. ${ }^{2}$, José Gerardo León Chimbolema. ${ }^{3} \&$ Eliana Soraya Sánchez Moreano. ${ }^{4}$

Recibido: 19-03-2019 / Revisado: 27-04-2019 /Aceptado: 28-05-2019/ Publicado: 05-06-2019

\begin{abstract}
DOI: https://doi.org/10.33262/cienciadigital.v3i2.6.574

The water resources with their catchment and supply systems of the Parish of CubijiesEcuador constitute a primary source of community participation and social management in a historic struggle for their conservation and use.

Based on the analysis of environmental sustainability, supply categories, regulation, cultural support and structure of the social fabric and applying the methods of economic valuation with inferential analysis, the link between an ecosystem service and the water resource was identified, thus defining the willingness to pay for the management, use and preservation of the resource. The factors that modified the perception of the capacity of the ecosystems were: level of education, gender and community, in parallel it was estimated that the environmental economic value of the water resource in the study area was directly related according to agricultural and domestic use. The water resource has a noticeable incidence within the economy and the local wellbeing, consequently actions must be undertaken for its conservation and protection.
\end{abstract}

Keywords: Hidric resource, Economic valuation of the water resource, socioeconomic evaluation, willingness to pay, community water management

1 Escuela Superior Politécnica de Chimborazo - ESPOCH, Facultad de Ciencias, Riobamba, Ecuador sofia.godoy@espoch.edu.ec

2 Escuela Superior Politécnica de Chimborazo - ESPOCH, Facultad de Ciencias, Riobamba, Ecuador andres.beltran@espoch.edu.ec

3 Escuela Superior Politécnica de Chimborazo - ESPOCH, Facultad de Ciencias, Riobamba, Ecuador gerardo.leon@espoch.edu.ec

4 Escuela Superior Politécnica de Chimborazo - ESPOCH, Facultad de Ciencias, Riobamba, Ecuador eliana.ssm.16@gmail.com 


\section{Resumen.}

Los recursos hídricos con sus sistemas de captación y abastecimiento de la Parroquia Cubijíes-Ecuador constituyen una fuente primaria de participación comunitaria y gestión social en una lucha histórica para su conservación y uso. Con base en el análisis de sostenibilidad ambiental, categorias de abastecimiento, regulación, de soporte culturales y estructura del tejido social y aplicando los métodos de valoración económica con análisis inferencial se identificó el vínculo existente entre un servicio ecosistémico y el recurso hídrico, definiendo así la disposición a pagar para el manejo, aprovechamiento y preservación del recurso. Los factores que modificaron la percepción de la capacidad de los ecosistemas fueron: nivel de instrucción, género y comunidad, paralelamente se estimó que el valor económico ambiental del recurso hídrico en la zona de estudio se relacionó directamente conforme al uso agrícola y doméstico. El recurso hídrico tiene una incidencia notoria dentro de la economía y el bienestar local, en consecuencia se deben emprender acciones para su conservación y protección.

Palabras claves: Recurso hídrico, Valoración económica del recurso hídrico, evaluación socioeconómica, disposición a pagar, gestión comunitaria del agua

\section{INTRODUCCIÓN}

Los servicios ecosistémicos son todos los beneficios que los seres humanos reciben de los ecosistemas, como resultado de su estructura y del desarrollo de funciones y/o procesos naturales (Fagerholm, Käyhkö, Ndumbaro , \& Khamis M, 2012). Dentro de los servicios ecosistémicos, aquellos relacionados con el agua son los de mayor importancia para el bienestar humano. (Hackbart, De Lima, \& Dos Santos, 2017), porque contribuyen a la subsistencia y al desarrollo económico de la sociedad (Grizzetti et al., 2016).

Conforme el Plan Nacional de Gestión del Agua del Ecuador se articulan acciones a favor de la conservación y protección de este recurso. Dentro de sus ejes temáticos destacan: calidad del agua, balances de oferta y demanda, costos y evaluación económica, eficiencia y tarifas (SENAGUA, 2011). En tal virtud, la valoración económica ambiental con el análisis del agua constituye una herramienta fundamental para su gestión, el pago por los servicios hídricos reduce la presión sobre el escaso presupuesto de los Gobiernos Autónomos Descentralizados (GADs) que desean invertir en programas de gestión integral (Asamblea Nacional de la República del Ecuador, 2015).

La parroquia de Cubijíes y su cabecera parroquial se encuentra situada en la provincia de Chimborazo, zona 3 de planificación del Ecuador. Comprende las comunidades de Socorro, San Jerónimo de Porlón y San Clemente. El recurso hídrico de la parroquia está compuesta por las fuentes de los Ríos: Guano, Chambo, vertientes propias de la parroquia y Vertientes externas (GADPR Cubijíes, 2014). 
En la parroquia de Cubijíes, el agua procedente de vertientes naturales y de los cauces de los ríos Chambo y Guano han permitido el desarrollo de actividades cotidianas y productivas en el sector (GADPR Cubijíes, 2015). El manejo y uso sostenible de los recursos hídricos debe considerar todos los servicios potenciales, razón por la cual, la identificación de los servicios hídricos contribuirá en la posterior evaluación de escenarios referentes al aprovechamiento o explotación de las fuentes hídricas (Grizzetti et al., 2016). El objetivo principal de la investigación se centró en la evaluación socioeconómica de la disposición a pagar por aprovechamiento, manejo y preservación del recurso hídrico en la parroquia rural Cubijíes, para la propuesta de políticas y estrategias que sirvan como referencia al GAD parroquial en su próxima actualización del Plan de Desarrollo y Ordenamiento Territorial.

\section{DESARROLLO}

\section{Marco teórico Referencial:}

En Ecuador estudios de valoración ambiental del recurso hídrico han sido realizados principalmente en la parte sur del territorio con la finalidad de conservar y proteger las fuentes hídricas, destacando entre ellas: Valoración socio-económica y ambiental del recurso hídrico de la microcuenca Atacuri, parroquia de Santiago - Provincia de Loja que permitió calcular el costo real del agua y los valores de captación y restauración además de estimar su valor como insumo para la producción mediante un análisis de demanda (Villavicencio (2008). En los cantones Célica y Espíndola de la misma provincia se realizó la valoración ambiental del recurso hídrico para la conservación de las microcuencas de Quillusara y Jorupe aplicando el método de valoración contingente para la recopilación de datos socio demográficos, de acceso a la información y de interés ambiental, que permitieron estimar la DAP por la conservación de las fuentes hídricas (Jaramillo y Coronel, 2013). En la ciudad de Catacocha, provincia de Loja, mediante el método de valoración contingente se estimó la disposición a pagar para la protección de las microcuencias (Armijos, 2013). Bajo las mismas características en la cuenca del río Tomebamba en la provincia de Azuay, se determinó la disposición a pagar la por el método contingente (Roldán, 2016).

Entre los estudios realizados en la provincia de Chimborazo destacan: la valoración económica de bienes y servicios hídricos y su incidencia en el sector productivo de la parroquia Quimiag (García y Jiménez, 2012). La valoración del servicio hidrológico de la cuenca del río Cebadas en el que fueron determinadas la oferta, la demanda y el índice de escasez hídrica del sector (Guambo et al., 2016). En la Parroquia Licto se estableció el valor económico ambiental para el agua de riego de la Junta General de Guargualla para el período 2012- 2016, conjuntamente con un modelo estadístico que definió la disposición a pagar en función de la disponibilidad del recurso, la diversidad en la zona y las características socio económicas (Remache, 2017). La valoración económica ambiental pretende asignar valores 
cuantitativos a los servicios ecosistémicos, independientemente de que éstos tengan un precio en el mercado (Tomasini, 2015, p. 1). El principal objetivo de la valoración ambiental a partir del análisis de la disposición a pagar es contribuir en la toma de decisiones para el manejo y la gestión de los recursos naturales, considerando la eficiencia económica total de los distintos usos (Tomasini, 2015, pp. 2-3), estima compensaciones en caso de producirse una alteración en el ambiente y destaca la importancia de los ecosistemas en las sociedades (Dias Carrilho y de Almeida Sinisgalli, 2018, p. 1).

\section{Marco metodológico:}

Investigación descriptiva y correlacional, con un diseño no experimental transversal. La población de estudio considerada fue sustentada con el método exponencial de proyección poblacional para el año 2018: con una población inicial de 2514 habitantes y una tasa de crecimiento anual de 1,45\% según el Instituto Nacional de Estadísticas y Censos del Ecuador (INEC).

Empelando técnicas de recolección de datos sustentados principalmente en encuestas, entrevistas semi estructuradas y grupos focales con los actores representativos de la sociedad, se consideraron tres componentes metodológicos: 1) diagnóstico base de Cubijíes que incluyó: caracterización de las fuentes hídricas, sistemas de abastecimiento, oferta - demanda hídrica y dimensión política.

2) determinación de los servicios ecosistémicos del recurso hídrico: se aplicó la metodología propuesta por (Brown et al., 2014). :

- Identificación y consulta a las partes interesadas "stakeholders": GADPR de Cubijíes, juntas administradoras de agua y usuarios del recurso. El proceso de consulta se llevó a cabo empleando grupos focales representativos de la parroquia.

- Identificación de los servicios ecosistémicos: involucrando un proceso interactivo y de consulta continua, donde se establecieron preguntas claves e indicadores de uso.

3) evaluación de la disposición a pagar de los servicios a hídricos: Los ecosistemas que se evaluaron fueron ríos y vertientes, bosques y/o cubierta vegetal aledaña a las fuentes hídricas. Para el efecto se consideraron los siguientes componentes:

- Factores que modifican la percepción de la capacidad de los ecosistemas: comunidad, género y nivel de instrucción. Se determinó la distribución de las variables mediante el método de Kolmogorov Smirnov y se aplicaron pruebas no paramétricas como la prueba de Mann Whitney para el factor género y de Kruskal Walllis para los factores comunidad y nivel de instrucción.

- Estimación del valor económico ambiental y la disposición a pagar por el recurso hídrico: Con la selección y valoración de los servicios hídricos se empleó el Plan de Desarrollo y Ordenamiento Territorial (PDyOT) de la parroquia de Cubijíes como herramienta clave para la priorización de los servicios hídricos, definidos en función de los objetivos y metas políticas. 
Una vez priorizados los servicios hídricos, se precisó la metodología a implementarse para cada servicio hídrico:

Tabla 1: Métodos de valoración económica ambiental.

\begin{tabular}{ll}
\hline SERVICIOS HÍDRICOS & MÉTODO DE VALORACIÓN \\
Agua de riego & Método residual \\
Agua de consumo humano & Valoración Contingente \\
\hline
\end{tabular}

Método residual: se calculó el valor del agua de riego en función de la productividad agrícola y de los gastos asociados a esta actividad. Para cada especie cultivada en la parroquia se estimó la producción en términos monetarios (ingresos) mediante el producto entre superficie de cultivo, rendimiento y precio medio en el mercado. Para calcular el valor del agua de riego de los ingresos de la venta de la producción agrícola se restaron los gastos asociados a la mano de obra y a los insumos excluyendo al agua.

Método de valoración contingente: empleado para calcular el valor del agua de consumo humano a partir de encuestas estructurada en 3 partes componentes;: datos generales del encuestado, simulación del escenario que se oferta, disposición a pagar.

Con estadística descriptiva (porcentajes y frecuencias) se procesó la información recopilada en las encuestas en torno a la situación actual del recurso hídrico, su conservación y la disposición a pagar (DAP). Se requirió también de un análisis estadístico inferencial de la DAP, por ello se aplicó la prueba Chi Cuadrado, adicionalmente se calculó el coeficiente de contingencia para verificar el grado de correlación de las variables.

\section{Análisis de los resultados:}

1) El Diagnóstico base de Cubijíes:

- Fuentes hídricas: El recurso hídrico de la parroquia de Cubijíes está compuesto por las siguientes fuentes: río Guano, río Chambo, vertientes propias de la parroquia y vertientes externas. En las comunidades de la parroquia de Cubijíes existe una distribución desigual del agua; las vertientes se concentran en ciertos lugares, sus caudales son variables y han disminuido con el paso del tiempo.

Solo una parte del caudal de las vertientes internas está concesionado para riego y servicios domésticos, mientras que el caudal restante se encuentra irrigando de forma permanente los humedales naturales de la parroquia, y los excedentes se usan para riego parcelario. Los excedentes de las vertientes externas desembocan en ríos cercanos.

\section{Sistemas de abastecimiento de agua de consumo humano:}

El sistema de abastecimiento de agua de consumo humano es diferente para cada comunidad, y la cobertura de este servicio es deficiente; el $72 \%$ de las familias se abastecen de los sistemas comunales (GADPR Cubijíes, 2014), mientras que el porcentaje restante emplea 
agua suministrada por tanqueros o la recogen en recipientes pequeños de las vertientes cercanas. Los principales sistemas de abastecimiento en la parroquia son:

Tabla 2: Principales sistemas de abastecimiento en la parroquia de Cubijíes.

\begin{tabular}{|c|c|c|}
\hline $\begin{array}{c}\text { SISTEMA DE } \\
\text { ABASTECIMIENTO }\end{array}$ & EXTENSIÓN TERRITORIAL & DESCRIPCIÓN \\
\hline $\begin{array}{l}\text { Sistema 1: Vertiente el } \\
\text { Ejido y Tobías Chávez }\end{array}$ & $\begin{array}{l}\text { Cabecera Parroquial, exceptuando al } \\
\text { barrio Bactús. }\end{array}$ & $\begin{array}{l}\text { Tratamiento: } \\
\text { (automática). } \\
\text { Distribución: Por gravedad. }\end{array}$ \\
\hline $\begin{array}{l}\text { Sistema 2: Vertientes } \\
\text { de Balcashi }\end{array}$ & $\begin{array}{l}\text { Comunidad del Socorro y ciertos } \\
\text { domicilios de la Comunidad de San } \\
\text { Jerónimo de Porlón }\end{array}$ & $\begin{array}{l}\text { Tratamiento: Cloración (manual). } \\
\text { Distribución: Por gravedad }\end{array}$ \\
\hline $\begin{array}{l}\text { Sistema 3: Vertientes } \\
\text { "EI Vergel" }\end{array}$ & $\begin{array}{l}\text { Comunidad San Clemente y gran } \\
\text { parte de la Comunidad de San } \\
\text { Jerónimo de Porlón }\end{array}$ & $\begin{array}{l}\text { Tratamiento: Cloración (manual). } \\
\text { Distribución: Bombeo (el sistema } \\
\text { dispone varias bombas: hidráulicas, } \\
\text { eléctrica y solar). }\end{array}$ \\
\hline
\end{tabular}

Análisis de oferta y demanda: Los principales usos del agua en la parroquia de Cubijíes son: doméstico y agrícola, como se observa en la siguiente tabla:

Tabla 3: Demanda hídrica en la parroquia de Cubijies.

\begin{tabular}{|c|c|c|}
\hline Uso & Valor & Unidad \\
\hline \multicolumn{3}{|l|}{ Doméstico } \\
\hline Dotación o consumo promedio & $135,37 *$ & L/hab d \\
\hline Número de habitantes & 2823 & hab \\
\hline Consumo mensual & 11464,49 & $\mathrm{~m}^{3}$ \\
\hline Consumo anual & 137573,82 & $\mathrm{~m}^{3}$ \\
\hline \multicolumn{3}{|l|}{ Agrícola } \\
\hline Área cultivada & 162 & ha \\
\hline $\begin{array}{l}\text { Consumo medio de los cultivos } \\
\text { predominantes en la zona }\end{array}$ & 6500 & $\mathrm{~m}^{3} / \mathrm{ha}$ \\
\hline Consumo total & 1053000,00 & $\mathrm{~m}^{3}$ \\
\hline Volumen total de agua empleado (anual) & 1190573,82 & $\mathbf{m}^{3}$ \\
\hline
\end{tabular}


Conforme a la tabla 3, la actividad agrícola demanda un volumen mayor de agua con relación al uso doméstico. La demanda hídrica total varía en función del tiempo; su cálculo depende de factores como el crecimiento poblacional y la expansión de la superficie destinada para cultivos. La oferta hídrica de la parroquia de Cubijíes depende del agua propia (procedente de los fenómenos de precipitación) e importada (caudal de ríos y vertientes externas):

Tabla 4: Oferta Hídrica en la parroquia de Cubijíes.

\begin{tabular}{lll}
\hline \multirow{2}{*}{$\begin{array}{lll}\text { Agua } \\
\text { propia }\end{array}$} & Precipitación Anual $\left(\mathrm{m}^{3} / \mathrm{m}^{2}\right)$ & 0,40 \\
\cline { 2 - 3 } & Oferta anual $\left(\mathrm{m}^{3}\right)$ & 1195000,00 \\
& Vertientes $\quad($ Volumen anual: & 47800,00 \\
& $\left.\mathrm{~m}^{3}\right)$ & 192844,80 \\
Agua & Ríos (Volumen anual: $\left.\mathrm{m}^{3}\right)$ & 9331200,00 \\
importada & Volumen total importado & 9524044,80 \\
Oferta total para la parroquia $\left(\mathrm{m}^{3}\right)$ & $\mathbf{1 0 0 0 2 0 4 4 , 8 0}$ \\
\hline
\end{tabular}

El volumen de agua propia es inferior al volumen de agua importada, en tal virtud, las aportaciones externas son de vital importancia para la parroquia de Cubijíes ya que abastecen las comunidades: Socorro, San Clemente y San Jerónimo de Porlón. En función de la oferta y la demanda hídrica, se calculó el índice de escasez:

$$
I E=\frac{1190573,82 \mathrm{~m}^{3}}{10002044,80 \mathrm{~m}^{3}} \times 100 \%=\mathbf{1 1}, \mathbf{9 0} \%
$$

Conforme al valor obtenido en el índice de escasez, la presión sobre el recurso hídrico es moderado (10 al 20\%), en consecuencia el agua puede limitar el desarrollo económico (principalmente la productividad agrícola) de la zona de estudio; siendo necesario emprender acciones a favor de la gestión y el monitoreo de las fuentes hídricas.

\section{Dimensión política:}

Los niveles de organización en torno al recurso hídrico en el caso del agua de consumo humano, en las Comunidades de Socorro, San Clemente y Porlón, el recurso es administrado por las Juntas de Agua de cada sistema En la cabecera parroquial quien asume las funciones es el GAD Parroquial. Con relación al agua de riego, en la cabecera parroquial existe una junta independiente; mientras que en las comunidades restantes las juntas de los reservorios están bajo la rectoría de la Junta General de Regantes del Chambo-Guano. 
2) Determinación de los servicios ecosistémicos del recurso hídrico:

Los resultados de las encuestas aplicadas a los actores principales permitieron identificar si los servicios ecosistémicos propuestos son importantes para la parroquia. Conforme al porcentaje de respuestas positivas se determinó que la mayor parte de los servicios hídricos son importantes para la población de la parroquia de Cubijíes, a excepción de los rituales culturales o espirituales. Los servicios hídricos de aprovisionamiento presentaron el $100 \%$ de respuestas positivas debido a que la población reconoce y valora principalmente servicios ecosistémicos tangibles. Los servicios hídricos de regulación y soporte exhibieron porcentajes variables dado que no todos los actores encuestados identificaron el vínculo existente entre el recurso hídrico y el servicio ecosistémico presentado:

Tabla 6: Resultados del proceso de consulta a los actores principales

\begin{tabular}{|c|c|c|c|}
\hline \multirow[t]{3}{*}{ Categoría } & \multirow{3}{*}{\multicolumn{2}{|c|}{ Servicio ecosistémico }} & Importancia \\
\hline & & & resp. \\
\hline & & & positivas) \\
\hline \multirow{4}{*}{ Aprovisionamiento } & Agua para consumo human & & 100 \\
\hline & Agua para riego & & 100 \\
\hline & Regulación hídrica & & 28,6 \\
\hline & Retención de nutrientes & & 14,3 \\
\hline \multirow[t]{2}{*}{ Regulación } & Mantenimiento del clima & & 71,4 \\
\hline & Mantenimiento & la & 57,1 \\
\hline \multirow[t]{4}{*}{ De soporte } & biodiversidad & & \\
\hline & Control de plagas & & 57,1 \\
\hline & Productividad agroforestal & & 57,1 \\
\hline & Rituales culturales & $\mathrm{y} / \mathrm{o}$ & 0 \\
\hline \multirow[t]{3}{*}{ Cultural } & espirituales & & \\
\hline & Recreación & & 57,1 \\
\hline & Belleza escénica & & 14,3 \\
\hline
\end{tabular}

De la categoría de servicios ecosistémicos culturales, la recreación y belleza escénica reportaron porcentajes variables; mientras que los rituales culturales y espirituales presentaron un porcentaje de 0 , debido a que en el lugar de estudio no existen fuentes hídricas destinadas o vinculadas a ese fin, consiguientemente este aspecto no se consideró en la evaluación posterior.

3) Evaluación de la disposición a pagar de los servicios a hídricos:

- Factores que modifican la percepción de la capacidad de los ecosistemas: comunidad, género y nivel de instrucción: 
Los factores que fueron objeto de análisis en esta sección fueron: Físicos o geográficos y sociales como lo son el nivel de instrucción y género.

Tabla 7: Factores que modifican la percepción de la potencialidad de los ecosistemas en la provisión de Servicios ecosistémicos

\begin{tabular}{|c|c|c|c|c|}
\hline FACTOR & ECOSISTEMA & $\begin{array}{l}\text { CATEGORÍA } \\
\text { DE SE }\end{array}$ & SERVICIO HÍDRICO & SIGNIFICANCIA \\
\hline \multirow{8}{*}{ Comunidad } & \multirow{4}{*}{$\begin{array}{l}\text { Acuático: Ríos y } \\
\text { vertientes }\end{array}$} & De regulación & Mantenimiento del clima & 0,028 \\
\hline & & \multirow[t]{2}{*}{ De soporte } & $\begin{array}{lll}\text { Conservación } & \text { de } & \text { la } \\
\text { Biodiversidad } & & \end{array}$ & 0,004 \\
\hline & & & Productividad Agroforestal & 0,006 \\
\hline & & Cultural & Belleza escénica & 0,000 \\
\hline & \multirow{4}{*}{$\begin{array}{l}\text { Bosques y/o cobertura } \\
\text { vegetal }\end{array}$} & De provisión & Agua de riego & 0,002 \\
\hline & & De regulación & Retención de nutrientes & 0,027 \\
\hline & & De soporte & Productividad Agroforestal & 0,023 \\
\hline & & Cultural & Belleza escénica & 0,000 \\
\hline \multirow{5}{*}{$\begin{array}{l}\text { Nivel de } \\
\text { instrucción }\end{array}$} & \multirow{4}{*}{$\begin{array}{l}\text { Acuático: Ríos y } \\
\text { vertientes }\end{array}$} & \multirow[t]{2}{*}{ De regulación } & Retención de nutrientes & 0,004 \\
\hline & & & Mantenimiento del clima & 0,032 \\
\hline & & De soporte & $\begin{array}{lll}\begin{array}{l}\text { Conservación } \\
\text { Biodiversidad }\end{array} & \text { de } & \text { la }\end{array}$ & 0,018 \\
\hline & & Culturales & Recreación & 0,012 \\
\hline & $\begin{array}{l}\text { Bosques y/o cobertura } \\
\text { vegetal }\end{array}$ & De regulación & Retención de nutrientes & 0,002 \\
\hline \multirow[b]{2}{*}{ Género } & $\begin{array}{l}\text { Acuático: Ríos y } \\
\text { vertientes }\end{array}$ & De soporte & Productividad Agroforestal & 0,030 \\
\hline & $\begin{array}{l}\text { Bosques y/o cobertura } \\
\text { vegetal }\end{array}$ & De soporte & $\begin{array}{lll}\begin{array}{l}\text { Conservación } \\
\text { Biodiversidad }\end{array} & \text { de } & \text { la }\end{array}$ & 0,050 \\
\hline
\end{tabular}

En función a los resultados (significancia $\rightarrow$ p) de las pruebas estadísticas empleadas, existe una diferencia entre las medias correspondientes en las cuatro comunidades. Los factores sociales condicionan los resultados obtenidos a partir del componente demográfico, siendo el de mayor incidencia el nivel de instrucción. Diversos estudios han demostrado que variables como: el género, la edad y el nivel de instrucción modifican la percepción en torno a la importancia y provisión de los servicios ecosistémicos (Affek \& Kowalska, 2017, p. 193; Allendorf \& Yang, 2013, pp. 191-192; Pettinotti et al., 2018, pp. 301- 303). La edad fue otro factor que modifica la percepción, sin embargo, las variaciones en torno a la edad son una consecuencia inmediata del grado de conocimiento de los individuos que está ligado con la escolaridad y la familiaridad con el entorno, es así que en el presente estudio no se contempló al factor edad (Allendorf y Yang, 2013, p. 192). Los servicios hídricos culturales reflejan que los individuos con grado académico superior otorgaron puntajes altos a la capacidad de los ríos y vertientes en actividades de recreación ( Affek y Kowalska, 2017, p. 188) Los encuestados con un mejor nivel de escolaridad atribuyen potenciales altos a servicios ecosistémicos culturales. 
Tabla 8: Medias del potencial de los ecosistemas en función del género.

\begin{tabular}{|c|c|c|c|}
\hline & & GÉNERO & \\
\hline $\begin{array}{l}\text { SERVICIO } \\
\text { HÍDRICO }\end{array}$ & & Femenino & Masculino \\
\hline $\begin{array}{l}\text { Acuático: } \\
\text { Agroforestal }\end{array}$ & Prod. & 6,74 & 7,20 \\
\hline $\begin{array}{l}\text { Bosque: } \\
\text { Biodiversidad }\end{array}$ & Cons. & 6,77 & 7,39 \\
\hline
\end{tabular}

Los valores medios del potencial de los bosques y la cobertura vegetal en la conservación de la biodiversidad, y de los ecosistemas acuáticos en la productividad agroforestal son mayores en el caso del género masculino; el hombre desempeña un papel importante dentro de la gestión y el manejo de los bosques, aspecto que contribuye en el entendimiento de sus funciones y sus vínculos con otros ecosistemas.

Tabla 9: Medias del potencial de los ecosistemas en las comunidades.

\begin{tabular}{lrllll}
\hline \multicolumn{5}{l}{$\begin{array}{l}\text { COMUNICIO } \\
\text { HÍDRICO }\end{array}$} & \multicolumn{3}{l}{$\begin{array}{l}\text { Cab. } \\
\text { Parroquial }\end{array}$} & Socorro & Porlón & $\begin{array}{l}\text { San } \\
\text { Clemente }\end{array}$ \\
\hline $\begin{array}{l}\text { Acuático: } \\
\text { Clima }\end{array}$ & Mant. & 7,20 & 6,25 & 7,14 & 6,42 \\
$\begin{array}{l}\text { Acuático: } \\
\text { Biodiversidad }\end{array}$ & & 7,57 & 6,39 & 7,36 & 6,99 \\
$\begin{array}{l}\text { Acuático: } \\
\begin{array}{l}\text { Agroforestal } \\
\text { Acuático: }\end{array}\end{array}$ & Prod. & 7,47 & 6,36 & 7,36 & 6,77 \\
$\begin{array}{l}\text { escénica } \\
\text { Bosque: }\end{array}$ & Belleza & 7,75 & 5,86 & 7,79 & 7,14 \\
$\begin{array}{l}\text { Bosque: } \\
\text { Nutrientes }\end{array}$ & Ret. & 5,92 & 6,01 & 8,83 & 7,42 \\
$\begin{array}{l}\text { Bosque } \\
\begin{array}{l}\text { Agroforestal } \\
\text { Bosque: }\end{array}\end{array}$ & Prod. & 7,28 & 6,73 & 7,36 & 4,67 \\
escénica & Belleza & 7,94 & 6,39 & 8,00 & 7,07 \\
\hline
\end{tabular}

Las medias del potencial de los ecosistemas varían en función de las comunidades analizadas, debido a que las percepciones dependen del componente social que es heterogéneo de una comunidad a otra.

- Estimación del valor económico ambiental y la disposición a pagar por el recurso hídrico: 
Estimación del valor económico ambiental y disposición a pagar por agua de riego: El ingreso total de la producción agrícola es de 839079,71 dólares, cantidad que puede variar como consecuencia del rendimiento de los cultivos a nivel de campo que es afectado por fenómenos como las heladas y las sequías (disponibilidad de agua). Por otra parte, los gastos asociados a las actividades agrícolas incluyen: preparación del terreno (arado), siembra, mantenimiento de los cultivos (fertilización, control de plagas y mano de obras para deshierba, aporque, etc.) y cosecha (mano de obra); adicionalmente los agricultores de la zona realizan el pago de la tarifa volumétrica y el fondo del agua en la Junta de Regantes del Chambo-Guano, fondos destinados para el mantenimiento del sistema de distribución del agua de riego y el cuidado de las fuentes hídricas respectivamente. En función al ingreso total y a los gastos no asociados al agua (mano de obra, maquinaria y otros insumos) se estimó el valor del agua de irrigación, tal como se observa en la tabla 10:

Tabla 10: Estimación del valor del agua de riego (método residual)

\begin{tabular}{|c|c|}
\hline & CANTIDAD \\
\hline $\begin{array}{l}\text { a. Ingresos de la actividad agrícola } \\
(\$)\end{array}$ & 839079,71 \\
\hline b. Gastos no asociados al agua $(\$)$ & 296360,00 \\
\hline Tarifas asociadas al agua & \\
\hline c. Tarifa volumétrica $(\$ / h a)$ & 35,00 \\
\hline d. Fondo del agua $(\$ / h a)$ & 4,00 \\
\hline Valor económico del agua $(a-b)$ & 542719,71 \\
\hline
\end{tabular}

Acorde al valor económico obtenido para el agua de riego se identifica que este recurso es importante para el desenvolvimiento de las actividades productivas del sector. Por lo tanto, en caso de que el volumen de agua disminuya, se limitaría significativamente la productividad agrícola.

Estimación del valor económico ambiental y disposición a pagar por agua de consumo:

Conforme al método contingente y los resultados de las encuestas empleadas, el 86,69\% de los encuestados (293 individuos) aceptó pagar un valor adicional para la conservación y protección de las fuentes hídricas, mientras que el 13,31\% (45 individuos) se negó. De aquellos quienes aceptaron pagar un valor adicional para la conservación y protección de las fuentes hídricas, 42,90\% asignaron un valor mensual de 0,50 - 1 dólares a la conservación de fuentes hídricas, 36,10\% indicaron que cancelarían 0,01 a 0,50 dólares, y 6,8\% optaron por el valor de 1,50 a 2 dólares, finalmente un 0,9\% escogieron el rango de 1 a 1,50 dólares. Por otra parte, el porcentaje restante no escogió ninguna respuesta ya que se negaron a pagar un valor adicional. 
No se estimó el valor de no uso con base en los resultados del proceso de consulta a actores sociales, la tendencia general fue no asignar un valor monetario al servicio ecosistémico pese a que éstos fueron identificados, en sí esto se debe a que la población paga únicamente por bienes tangibles.

Estadística inferencial en torno a la DAP.

Tabla 11: Dependencia entre las variables analizadas y la DAP.

\begin{tabular}{|c|c|c|c|}
\hline \multirow[b]{2}{*}{ Dependencia } & \multirow[b]{2}{*}{ Variable independiente } & \multicolumn{2}{|c|}{ Var. Dependiente: DAP } \\
\hline & & $\begin{array}{l}\text { Significancia } \\
\text { asintótica }\end{array}$ & $\begin{array}{l}\text { Coeficiente de } \\
\text { contingencia. }\end{array}$ \\
\hline & Edad & 0,001 & 0,297 \\
\hline \multirow{5}{*}{$S I$} & Instrucción & 0,033 & 0,337 \\
\hline & Ingresos & 0,010 & 0,237 \\
\hline & Incidencia del estado de & 0,005 & 0,205 \\
\hline & $\begin{array}{l}\text { las f. hídricas y la } \\
\text { cobertura en la } \\
\text { disponibilidad de agua. }\end{array}$ & & \\
\hline & $\begin{array}{l}\text { Nivel de importancia de } \\
\text { la conservación de las } \\
\text { fuentes hídricas }\end{array}$ & 0,018 & 0,228 \\
\hline \multirow{4}{*}{ NO } & Género & 0,322 & 0,117 \\
\hline & Uso prioritario & 0,880 & 0,105 \\
\hline & Tarifa actual & 0,404 & 0,189 \\
\hline & $\begin{array}{l}\text { Disminución de la } \\
\text { cantidad de agua }\end{array}$ & 0,146 & 0,141 \\
\hline
\end{tabular}

La tabla 11 indica que existe dependencia entre la DAP y variables socioeconómicas como: la edad, el nivel de instrucción y los ingresos mensuales; también se relaciona con la incidencia del estado de las fuentes hídricas, la disponibilidad de agua para la cobertura vegetal y el nivel de importancia de la conservación.(García y Jiménez, 2012; Remache, 2017).El género no fue una variable condicionante en la DAP de los pobladores de la parroquia de Cubijíes, se presume que esta diferencia se debe a que en los últimos años, la sociedad en general ha ido tomando conciencia en cuanto a la conservación de los recursos, eliminándose el concepto de que la mujer es la encargada de la gestión del agua.

\section{Conclusiones:}

- La línea base del lugar principalmente a través de la oferta y demanda del recurso hídrico identificó que el componente hidrográfico de la parroquia de Cubijíes está constituido por 12 vertientes (10 internas y 2 externas) que requieren de la aplicación de programas de gestión que contemplen su conservación y la protección de los puntos de embalse y la cobertura vegetal cercana. 
- Los servicios ecosistémicos identificados contemplan las categorías de abastecimiento o provisión (agua de consumo humano y de riego), de regulación (retención de nutrientes, regulación hídrica y mantenimiento del clima), de soporte (conservación de la biodiversidad, control de plagas y productividad agroforestal) y culturales (rituales culturales y espirituales, belleza escénica y recreación), descartándose de ésta última los rituales culturales y espirituales al no haber fuentes vinculadas a este fin en la parroquia.

- La evaluación de los servicios hídricos se determinó que los factores que modifican la percepción del potencial de los ecosistemas son la Comunidad, nivel de instrucción y género.

- La disposición a pagar por aprovechamiento y preservación del recurso hídrico en la parroquia Cubijíes representa en proporción mayoritaria la voluntad por pagar un valor adicional por la conservación de las fuentes hídricas equivalente a 0,54 \$ mensual. Por medio de análisis inferencial se determinó que la DAP depende de las siguientes variables: edad, instrucción, ingresos familiares, incidencia del estado de las fuentes hídricas y la cobertura vegetal en la cantidad de agua y el nivel de importancia de la conservación para el encuestado. El género no fue una variable condicionante en la DAP de los pobladores de la parroquia de Cubijíes, esto a casusa de que en los últimos años, la sociedad en general ha ido tomando conciencia respecto a la conservación de los recursos, eliminándose el concepto de que la mujer es la encargada de la gestión del agua.

- Los resultados obtenidos en este estudio son un referente para el reajuste de las tarifas del servicio de agua potable considerando que el valor ambiental se expresa en función de la disponibilidad a pagar por parte de los consumidores o beneficiarios, siendo ésta una de las competencias asignadas a los GADs con la finalidad de que éstos dispongan de recursos económicos destinados a la conservación de las fuentes y zonas de recarga hídrica

\section{Referencias Bibliográficas:}

Affek, A. N., \& Kowalska, A. (2017). Ecosystem potentials to provide services in the view of direct users. Ecosystem Services, 26, 183-196. https://doi.org/10.1016/j.ecoser.2017.06.017

Agudelo, R. (2005). El agua, recurso estratégico del siglo XXI. Scielo.

Allendorf, T. D., \& Yang, J. (2013). The role of ecosystem services in park-people relationships: The case of Gaoligongshan Nature Reserve in southwest China. Biological Conservation, 167, 187-193.https://doi.org/10.1016/j.biocon.2013.08.013

Armijos, L. (2013). Determinación de los costos de oportunidad y disposición de pagar (DAP) para la conservación del recurso hídrico en la ciudad de Catacocha. Universidad Católica de Loja, Loja. 
Asamblea Nacional de la República del Ecuador. (2015). Ley Orgánica de Recursos Hídricos, usos y aprovechamiento del agua. Ediciones Legales.

Brown, C., Reyers, B., Ingwall-King, L., Mapendembe, A., Nel, J., O Farrel, P., BowlesNewark, N. (2014). Measuring ecosystem services.

Cisneros, J., Alpízar, F., \& Madrigal, R. (2007). Valoración económica de los beneficios de protección del recurso hídrico bajo un esquema de pago por servicios ecosistémicos en Copán Ruinas, Honduras. Recursos Naturales y Ambiente, 51, 143-152.

Delgado, W. G. (2015). Gestión y valor del recurso hídrico. Revista Finanzas y Política Económica, 279-298.

https://doi.org/10.14718/revfinanzpolitecon.2015.7.2.4

Dias Carrilho, C., \& de Almeida Sinisgalli, P. A. (2018). Contribution to Araçá Bay management: The identification and valuation of ecosystem services. Ocean \& Coastal Management. https://doi.org/10.1016/j.ocecoaman.2018.03.023

Ellison, D., Morris, C. E., Locatelli, B., Sheil, D., Cohen, J., Murdiyarso, D., Sullivan, C. A. (2017). Trees, forests and water: Cool insights for a hot world. Global Environmental Change, 43, 51-61.

https://doi.org/10.1016/j.gloenvcha.2017.01.002

Fagerholm, N., Käyhkö, N., Ndumbaro, F., \& Khamis, M. (2012). Community stakeholders' knowledge in landscape assessments - Mapping indicators for landscape services. Ecological Indicators, 18, 421-433.

https://doi.org/10.1016/j.ecolind.2011.12.004

GADPR Cubijíes. (2014). Plan de Ordenamiento y Desarrollo Territorial de Cubijíes (20142019). Recuperado de https://www.usfq.edu.ec/programas_academicos/colegios/cociba/quitoambiente/temas_amb ientales/cambio_climatico/Documents/DC2AC1_atlas_ambiental_dmq_clima.pdf GADPR Cubijíes. (2015). Características de la Parroquia de Cubijíes. Recuperado de http://app.sni.gob.ec/snilink/sni/PORTAL_SNI/data_sigad_plus/sigadplusdiagnostico/0660 822960001_DIAGNOSTICO\%20POR\%20COMPONENTES_15-05-2015_23-14-21.pdf

García, N. S., \& Jiménez, C. del R. (2012). Valoración económica de Bienes y Servicios Ambientales del Recurso Hídrico de la Parroquia Quimiag y su incidencia en los sectores productivos en el período 2007-2009 (B.S. thesis). Riobamba: Universidad Nacional de Chimborazo, 2016.

Grizzetti, B., Lanzanova, D., Liquete, C., Reynaud, A., \& Cardoso, A. C (2016). Assessing water ecosystem services for water resource management. Environmental Science \& Policy, 61, 194-203.

https://doi.org/10.1016/j.envsci.2016.04.008

Hackbart, V., De Lima, G., \& dos Santos, R. (2017). Theory and practice of water ecosystem services valuation: where are we going? Ecosystem services, 23, 218-227. 
Guambo, A., Arguello, C., Zurita, G., \& Romero, J. (2016). El valor económico ambiental de los usuarios del servicio hidrológico de la cuenca del río Cebadas, Provincia de Chimborazo. Revista Sathiri, 11.

Jaramillo, R., \& Coronel, D. (2013). Valoración económica del recurso hídrico para la conservación de las microcuencas Quillusara en el cantón Célica y Jorupe en el Cantón Espíndola. (B.S. thesis). Universidad Católica de Loja, Loja. Recuperado de http://www.keneamazon.net/Documents/Publications/Virtual-Library/EconomiaDesarrollo/122.pdf

Remache, L. (2017). Valoración económica ambiental del recurso hídrico, y el beneficio para los usuarios de la junta general del sistema de riego Guargualla de la parroquia Licto cantón Riobamba provincia de Chimborazo, periodo 2012, 2016. (B.S. thesis). Universidad Nacional de Chimborazo, 2017.

Roldán, F. (2016). Valoración económica de recursos hídricos para el suministro de agua potable: El caso del Parque Nacional Cajas y la Cuenca del río Tomebamba. Universidad de Alicante. Recuperado de https://rua.ua.es/dspace/bitstream/10045/54425/1/tesis_roldan_monsalve.pdf SENAGUA. (2011). Plan Nacional de los Recursos Hídricos. Presentado en Seminario Taller, Quito.

Tomasini, D. (2015). Valoración económica del Ambiente. Revista Economía y Desarrollo. Villavicencio, C. A. (2008). Valoración socioeconómica y ambiental del recurso hídrico de la microcuenca Atacurí, parroquia Santiago, cantón Loja (B.S. thesis). 
PARA CITAR EL ARTÍCULO INDEXADO.

Godoy Ponce, S., Beltrán Dávalos, A., León Chimbolema, J., \& Sánchez Moreano, E. (2019).

Evaluación socioeconómica de disposición a pagar por aprovechamiento y preservación del recurso $\begin{array}{lllll}\text { hídrico en } \quad \text { Cubijíes. } & \text { Ciencia } & \text { 406-42.6), }\end{array}$ https://doi.org/10.33262/cienciadigital.v3i2.6.574

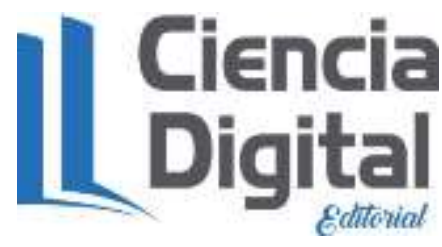

El artículo que se publica es de exclusiva responsabilidad de los autores y no necesariamente reflejan el pensamiento de la Revista Ciencia Digital.

El artículo queda en propiedad de la revista y, por tanto, su publicación parcial y/o total en otro medio tiene que ser autorizado por el director de la Revista Ciencia Digital.
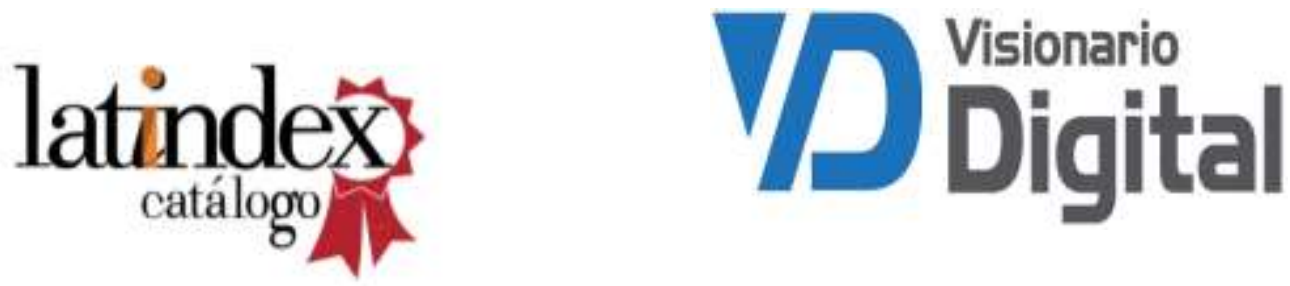